\title{
Bounded Risk Estimation of the Gamma Scale Parameter in a Purely Sequential Sampling Procedure
}

\author{
Eisa Mahmoudi ${ }^{\star}$, Ghahraman Roughani, Ashkan Khalifeh \\ Department of Statistics, Yazd University, Yazd, Iran
}

\section{ARTICLE INFO}

Article History

Received 06 Nov 2017

Accepted 24 May 2018

\section{Keywords}

Bounded risk estimation

Gamma distribution

Sequential estimation

Stopping variable

Two-stage sampling

Purely sequential procedure

\begin{abstract}
We consider the purely sequential procedure for estimating the scale parameter of a gamma distribution with known shape parameter, when the risk function is bounded by the known preassigned number. In this paper, we provide asymptotic formulas for the expectation of the total sample size. Also, we propose how to adjust the stopping variable so that the risk is uniformly bounded by a known preassigned number. In the end, the performances of the proposed methodology are investigated with the help of simulations and also by using a real data set.
\end{abstract}

(c) 2019 The Authors. Published by Atlantis Press SARL. This is an open access article distributed under the CC BY-NC 4.0 license (http://creativecommons.org/licenses/by-nc/4.0/).

\section{INTRODUCTION}

The problem of sequential estimation refers to any estimation technique for which the total number of observations used is not a degenerate random variable. In some statistical problems, especially in estimation fields, the sequential estimation must be used because no procedure based on fixed sample size can achieve the desired objective. Working with a sequential sampling procedure is necessary for some estimation problems with unknown parameters.

Sequential estimation of the scale parameter of a gamma distribution was first considered by Woodroofe [1], but this problem has not attracted attention from authors. Only Takada and Nagata [2] and Zacks and Khan [3] studied the confidence intervals of the mean and scale parameter of a gamma distribution. Also, Isogai and Uno [4] considered the sequential procedure for estimating the mean of a gamma distribution when the loss function is squared error plus linear cost.

Recently, Mahmoudi and Roughani [5] studied the two-stage sampling procedure for estimating the scale parameter of a gamma distribution with known shape parameter. They provided explicit formulas for the distribution and expected value of the stopping variable. Also, Roughani and Mahmoudi [6] continued and completed the study of the two-stage sampling procedure for estimating the scale parameter of a gamma distribution that have been proposed by Mahmoudi and Roughani [5]. They provided explicit formulas for the expected value and risk of the estimator of a gamma scale parameter, where the shape parameter is known. In spite of a little work has been done on gamma distribution, many investigators focused on exponential distribution. Starr and Woodroofe [7], Mukhopadhyay [8], Isogai and Uno [9], Mukhopadhyay [10], Mukhopadhyay and Datta [11], Uno et al. [12], Zacks and Mukhopadhyay [13,14], Mukhopadhyay and Pepe [15], Zacks [16], Mahmoudi and Lalehzari [17], Lalehzari et al. [18] and some others introduced many methods for sequential estimation of the scale parameter of an exponential distribution.

Survival and reliability analysis are two important scientific fields of study where the gamma distribution is most often used to model data. In survival analysis, variables such as lifespan of organisms as well as time till a treatment takes effect can be modelled with the gamma distribution. In reliability studies, lifespan of a system or systems components as well as chemical corrosion, e.g. can be modelled with the gamma distribution. The information gained by these two statistical models, is used to develop life insurance plans, pertinent drug information, warranty information, quality control information, etc. A parameter often studied in these fields is Mean Time to Failure (MTTF) that is very useful for systems used on a regular basis (see, e.g., Amero and Bayarri [19]; Choe and Shroff [20]). 
The study about the scale parameter of a gamma distribution with known shape parameter is important because of (i) one can check the robustness of an exponential distribution when the shape parameter in gamma distribution is known. (ii) To avoid very long computational time in detecting multiple change points in a gamma-distributed sample, previous change point detection algorithms usually assume a known shape parameter $\alpha$ (Killick and Eckley [21]). (iii) When modelling failure times with a known number of failures and missing values are present, the time between one failure and the last record is gamma with known shape. This happens often when data are recorded periodically and not after each failure. MTTF is often estimated as an exponential random variable, however in many cases, MTTF is modeled with a gamma distribution when the shape is known and not equal to one. Dopke [22] and Coit and Jin [23] discussed the estimation of the MTTF as a gamma random variable. The example given by Coit and Jin is when the time between each failure is not recorded. If there are $k$ failures in a span $t$, then the MTTF is gamma distributed with known shape $k$ and unknown scale. (iv) There are particular instances where the shape parameter is either known or can be assumed as known. These situations occur with modelling times and normal distribution; there are modelling times when the shape parameter is assumed known just as there are instances in the normal distribution when variance is assumed known. This can happen for a number of reasons; either there is so much historical evidence that the shape is consistent, there exists some mathematical theory for the shapes value, or the actual shape is of little concern as long as it is within reason. For example, Mareuil et al. [24] in precipitation models, discussed the actual unimportant of knowing the exact shape. They stated that since the data is right skewed, it is important to model the data with a low shape value. In this dissertation, they simply modelled precipitation intensity with $\alpha=2$. (v) One cannot reduce the case of $\operatorname{Gamma}(\alpha, 1)$ to that of $\operatorname{Gamma}(1,1)$ if $\alpha$ is arbitrary, if $\alpha=1 / 2$ then one could base the procedure on sums of two independent random variables and reduce the case to that of exponential jumps. But, this is not efficient and can be done only if $\alpha=1 / i$, for $i=1,2, \cdots$, (Zacks and Khan, [3], p. 298). For more explanation about this item, consider the arrival process where the random variable $S_{i}$ denotes the time until the $i$ th outcome is occurred, $X_{i}$ is the time between $i-1$ and $i$ th outcome and $N(t)$ as the Poisson process with rate $\lambda$ denotes the number of outcomes until time $t$. We know that $X_{i}=S_{i}-S_{i-1}$ has exponential distribution with mean $\beta=1 / \lambda, S_{i}=\sum_{j=1}^{i} X_{j} \sim \operatorname{Gamma}(i, \beta), i \in \mathbb{N}$ and $N(t) \sim$ Poisson $(\lambda t)$. The random variable $X_{i}, i \in \mathbb{N}$, with Gamma $(1, \beta)$ can be denoted as sum $X_{i}=\sum_{j=1}^{i} X_{i j}$, where $X_{i j}$ 's are independent random variables with Gamma $(1 / i, \beta)$ distribution.

In this paper, we consider the purely sequential procedure for estimating the scale parameter of a gamma distribution, with known shape parameter, when the risk function is bounded by the known preassigned number $\omega(>0)$. Also, we provide asymptotic formulas for the expected value of the total sample size, i.e., $E[N]$. We propose how to adjust the stopping variable $N$, so that the risk is uniformly bounded by a known preassigned number $\omega(>0)$. In the end, the performances of the proposed methodology are investigated with the help of simulations and by using a real data set.

The reminder of the paper is organized as follows:

In Section 2, we introduce the purely sequential procedure which is used in this paper. The expected value and bias of $N$ and $\hat{\beta}_{N}$ are calculated in Section 3. To investigate the results of the previous sections, a simulation study is carried out in Section 4 . In Section 5, the purely sequential procedure based on adjusted $N$, i.e., $N_{a d j}$, is introduced and its bias and expectation is obtained. We compare the two-stage and purely sequential procedures in Section 6. To investigate the results of this sequential problem, we use one application of a real data set in Section 7. Finally, Section 8 concludes the paper and discusses the results.

\section{PURELY SEQUENTIAL PROCEDURE}

Consider we observe a sequence of independent and identically distributed random variables $X_{1}, X_{2}, \cdots$ from a gamma distribution, with common probability density function

$$
f(x ; \alpha, \beta)=\frac{1}{\Gamma(\alpha) \beta^{\alpha}} x^{\alpha-1} e^{-\frac{x}{\beta}} I(x>0),
$$

where the scale parameter $\beta(>0)$ is unknown, but the shape parameter $\alpha(>0)$ is known.

Having observed $X_{1}, X_{2}, \cdots, X_{n}$, let $\beta$ is estimated by $\hat{\beta}_{n}=\frac{\sum X_{i}}{n \alpha}=\frac{\bar{X}_{n}}{\alpha}$ and the loss function in estimating $\beta$ by $\hat{\beta}_{n}$ is given by

$$
L\left(\hat{\beta}_{n}, \beta\right)=A\left(\hat{\beta}_{n}-\beta\right)^{2} \text {, }
$$

where $A$ is a positive known weight.

Our goal is to make the risk function associated with this loss function less than a preassigned number $\omega(>0)$, i.e.,

$$
A E\left[\left(\hat{\beta}_{n}-\beta\right)^{2}\right] \leq \omega .
$$

The risk is $R\left(\hat{\beta}_{n}, \beta\right)=E\left[A\left(\hat{\beta}_{n}-\beta\right)^{2}\right]=\frac{A \beta^{2}}{\alpha n}$, and this will be at most $\omega$ if $n \geq \frac{A \beta^{2}}{\alpha \omega}=n^{*} . n^{*}$ may not be an integer. It is called the optimal fixed sample size and remains unknown since $\beta$ is unknown. Takada [25] showed that no procedure based on fixed sample size can solve this problem. 
In the purely sequential procedure, $n^{*}$ is estimated successively in a sequential manner. One may begin with $m(\geq 2)$ observations and then continue to take one additional observation at a time, but terminate sampling when he has gathered enough observations. At each stage, he estimates $\beta$ and translates it to estimate $n^{*}$, then checks whether the sample size exceeds the estimate of $n^{*}$. As soon as the sample size exceeds the estimate of $n^{*}$, the sampling is terminated and final estimate of $\beta$ is offered.

The optimal sample size $n^{*}=\frac{A \beta^{2}}{\alpha \omega}$ is a function of unknown parameter $\beta$, hence we start with $X_{1}, X_{2}, \cdots, X_{m}$, as pilot sample observations following the gamma distribution in (1). Then, we will proceed with one additional observation at a time, and terminate sampling according to the stopping time

$$
N=\inf \left\{n \geq m ; n \geq \frac{A \bar{X}_{n}^{2}}{\alpha^{3} \omega}\right\}
$$

This procedure is worked as follows. In the initial stage, we compute $\bar{X}_{m}$ based on $X_{1}, X_{2}, \cdots, X_{m}$ and check whether $m \geq \frac{A \bar{X}_{m}^{2}}{\alpha^{3} \omega}$. Note that $\frac{A \bar{X}_{m}^{2}}{\alpha^{3} \omega}$ is an estimatorof $n^{*}$ at this stage. If $m \geq \frac{A \bar{X}_{m}^{2}}{\alpha^{3} \omega}$, then we stop sampling and our final sample size is $m$, i.e., $N=m$. But, if $m<\frac{A \bar{X}_{m}^{2}}{\alpha^{3} \omega}$ we take one additional observation $X_{m+1}$ and update the sample mean by $\bar{X}_{m+1}$ based on $X_{1}, X_{2}, \cdots, X_{m}, X_{m+1}$. Again, we check whether $m+1 \geq \frac{A \bar{X}_{m+1}^{2}}{\alpha^{3} \omega}$ as an estimate of $n^{*}$ at this stage. If $m+1 \geq \frac{A \bar{X}_{m+1}^{2}}{\alpha^{3} \omega}$ then sampling is terminated and $N=m+1$, else we must take another observation. This continues until we arrive at the sample size $n$ for the first time which is at least as large as the associated estimator of $n^{*}$, namely $\frac{A \bar{X}_{n}^{2}}{\alpha^{3} \omega}$. Hence, the final sample size is $n$, i.e, $N=n$. Finally, we use $X_{1}, X_{2}, \cdots, X_{N}$ and introduce $\hat{\beta}_{N}=\frac{\bar{X}_{N}}{\alpha}$ as a final estimator of $\beta$.

\section{THE EXPECTED VALUE AND BIAS OF $N$ AND $\hat{\beta}_{N}$}

If we consider (3), we find that the exact probability distribution of $N$ is hard to compute. For example, let $m=20$ and the sequential sampling stopped with $N=24$. This means that we did not stop with 20, 21, 22 or 23 observations, but we stopped with 24 observations. In other words, the event $\{N=24\}$ is the same as the event

$$
\left\{20<\frac{A \bar{X}_{20}^{2}}{\alpha^{3} \omega}, \cdots, 23<\frac{A \bar{X}_{23}^{2}}{\alpha^{3} \omega} \text { and } 24 \geq \frac{A \bar{X}_{24}^{2}}{\alpha^{3} \omega}\right\}
$$

and finding the probability of this event is very hard. But, we can compute the approximate expected value of $N$.

Let $G(x, \alpha, \beta)$ denotes the cumulative distribution function (c.d.f.) of a gamma distribution with shape parameter $\alpha$ and scale parameter $\beta$, i.e., $G(x, \alpha, \beta)=P(X \leq x)$ where $X \sim \operatorname{Gamma}(\alpha, \beta)$ and $\bar{G}(x, \alpha, \beta)=1-G(x, \alpha, \beta)$.

Theorem 3.1. (The first moment of $N$ ) Suppose that $m \alpha>2$ and $r>2$. If $n^{*}>m$ then

$$
\begin{aligned}
E(N)= & n^{*}-\frac{1}{\alpha}+\frac{1}{2}-2 \sum_{n=1}^{\infty}\left[\bar{G}\left(\frac{3}{2} n \alpha, n \alpha+1,1\right)\right. \\
& \left.-\frac{3}{2} \bar{G}\left(\frac{3}{2} n \alpha, n \alpha, 1\right)\right]+o(1),
\end{aligned}
$$

as $\omega \rightarrow 0$.

Proof. The proof of this theorem is a special case of Theorem 5.1 for $L_{0}=0$, presented in Section 5 of the paper.

From (4), we can obtain that $N$ is a bias estimator of $n^{*}$ and its bias is given by

$$
\begin{aligned}
\operatorname{Bias}\left(N, n^{*}\right)= & -\frac{1}{\alpha}+\frac{1}{2}-2 \sum_{n=1}^{\infty}\left[\bar{G}\left(\frac{3}{2} n \alpha, n \alpha+1,1\right)\right. \\
& \left.-\frac{3}{2} \bar{G}\left(\frac{3}{2} n \alpha, n \alpha, 1\right)\right]+o(1),
\end{aligned}
$$

as $\omega \rightarrow 0$. The bias is not always negative. Its negatively or positively depends on values of $\alpha$. The values of

$$
\sum_{n=1}^{\infty}\left[\bar{G}\left(\frac{3}{2} n \alpha, n \alpha+1,1\right)-\frac{3}{2} \bar{G}\left(\frac{3}{2} n, n \alpha, 1\right)\right]
$$


is positive for each values of $\alpha$ and is decreasing as a function of $\alpha$. Thus, the values of

$$
-2 \sum_{n=1}^{\infty}\left[\bar{G}\left(\frac{3}{2} n \alpha, n \alpha+1,1\right)-\frac{3}{2} \bar{G}\left(\frac{3}{2} n \alpha, n \alpha, 1\right)\right]
$$

is negative. Therefore, the bias of $N$, Bias $\left(N, n^{*}\right)$, may be negative or positive which depends on the values of $\alpha$. For example for $\alpha=2$, we have $\operatorname{Bias}\left(N, n^{*}\right)=-0.568$ and for $\alpha=4, \operatorname{Bias}\left(N, n^{*}\right)=0.0524$.

Theorem 3.2. The stopping time $N$ in (3), satisfies the second order asymptotic efficiency results, i.e., $E(N)-n^{*}=O(1)$.

Proof. For proof this property, it is enough to show that $\sum_{n=1}^{\infty} \bar{G}\left(\frac{3}{2} n \alpha, n \alpha, 1\right)<\infty$ and $\sum_{n=1}^{\infty} \bar{G}\left(\frac{3}{2} n \alpha, n \alpha+1,1\right)<\infty$.

For $X \sim \Gamma(n \alpha+1,1)$ and $t<1$, using Chernoff inequality, we have

$$
\begin{aligned}
\bar{G}\left(\frac{3}{2} n \alpha, n \alpha+1,1\right) & =P\left(X>\frac{3}{2} n \alpha\right) \\
& \leq \inf _{t<0} e^{-\frac{3}{2} n \alpha t} M_{X}(t)=\inf _{t<0} e^{-\frac{3}{2} n \alpha t}(1-t)^{-n \alpha-1},
\end{aligned}
$$

where $M_{X}(t)$ denotes the moment generating function of $X$ and exists for $t<1$. Using first derivative test, it is easy to check that the function $g(t)=e^{-\frac{3}{2} n \alpha t}(1-t)^{-n \alpha-1}$ attains its global minimum at $t=(0.5 n \alpha-1) / 1.5 n \alpha$ which is less than 1 for all $\alpha$. Hence,

$$
\begin{aligned}
\bar{G}\left(\frac{3}{2} n \alpha, n \alpha+1,1\right) & =P\left(X>\frac{3}{2} n \alpha\right) \\
& \leq \inf _{t>0} g(t)=\left(\frac{1.5 n \alpha}{n \alpha+1}\right)^{n \alpha+1} e^{-\frac{1}{2} n \alpha+1} \\
& \leq\left(\frac{1.5 n \alpha}{n \alpha}\right)^{n \alpha+1} \frac{e}{\sqrt{e}^{n \alpha}}=1.5 e\left(\frac{1.5}{\sqrt{e}}\right)^{n \alpha} .
\end{aligned}
$$

Thus, $\sum_{n=1}^{\infty} \bar{G}\left(\frac{3}{2} n \alpha, n \alpha+1,1\right)<\infty$, and hence, $\sum_{n=1}^{\infty} \bar{G}\left(\frac{3}{2} n \alpha, n \alpha, 1\right) \leq \sum_{n=1}^{\infty} \bar{G}\left(\frac{3}{2} n \alpha, n \alpha+1,1\right)<\infty$, since $1.5<\sqrt{e}$. The proof is completed.

Theorem 3.3. If $m \alpha>2$ and $r>2$ then the expectation of $\hat{\beta}_{N}$ is

$$
E\left(\hat{\beta}_{N}\right)=\beta-\frac{2 \beta}{\alpha n^{*}}+o(1)
$$

and its bias as a function of $\beta$ is given by

$$
\operatorname{Bias}\left(\hat{\beta}_{N}, \beta\right)=-\frac{2 \beta}{\alpha n^{*}}+o(1),
$$

as $\omega \rightarrow 0$.

Proof. The proof of this theorem is a special case of Theorem 5.2 for $L_{0}=0$, presented in Section 5 of the paper.

We can use the result of this theorem and introduce a corrected estimator of $\beta$ as follows:

$$
\hat{\beta}_{N}^{c}=\frac{\bar{X}_{N}}{\alpha}+\frac{2 \bar{X}_{N}}{\alpha^{2} N}=\frac{\bar{X}_{N}}{\alpha}\left(1+\frac{2}{\alpha N}\right) .
$$

This is an estimator with negligible bias and therefore, is better than $\hat{\beta}_{N}$.

\section{SIMULATION RESULTS}

To investigate the results of the previous sections, a simulation study is carried out. We considered $m=20, A=2, \alpha=1$ and 3.7 and $\beta=1,2, \cdots, 25$. For each set of values of $A, m, \alpha$ and $\beta$, we choose $\omega=2,1,0.5,0.25,0.1$ and 0.05 , so that $n^{*}$ was determined by $\frac{A \beta^{2}}{\alpha \omega}$. Then, for any combination of $m, \alpha, \beta$ and $\omega$, we ran $h=10000$ replications by letting R-program draws random samples from the assigned gamma population. Suppose that in the ith replication, we observe $n_{i}$ observations. Based on this data, the usual estimate of $\beta$ is $\hat{\beta}_{n_{i}}=\frac{\bar{x}_{n_{i}}}{\alpha}$ and its corrected estimate is $\hat{\beta}_{n_{i}}^{c}=\frac{\bar{x}_{n_{i}}}{\alpha}\left(1+\frac{2}{\alpha n_{i}}\right)$. 
To summarize the results, we use the following notations:

1. $\hat{E}(N)$ shows the simulated value of $E(N)$ and is computed by $\bar{n}=h^{-1} \sum_{i=1}^{h} n_{i}$.

2. $\hat{E}\left(\hat{\beta}_{N}\right)$ shows the simulated value of $E\left(\hat{\beta}_{N}\right)$ and is computed by $h^{-1} \sum_{i=1}^{h} \frac{\bar{x}_{n_{i}}}{\alpha}$.

3. The simulated bias of $\hat{\beta}_{N}$ is shown by $\hat{B}\left(\hat{\beta}_{N}, \beta\right)$ and its formula is $\hat{E}\left(\hat{\beta}_{N}\right)-\beta$.

4. The simulated risk of $\hat{\beta}_{N}$ is shown by $\hat{R}\left(\hat{\beta}_{N}, \beta\right)$ and its formula is $\bar{r}=A h^{-1} \sum_{i=1}^{h}\left(\frac{\bar{x}_{n_{i}}}{\alpha}-\beta\right)^{2}$.

5. $\hat{E}\left(\hat{\beta}_{N}^{c}\right)$ shows the simulated value of $E\left(\hat{\beta}_{N}^{c}\right)$ and is computed by $h^{-1} \sum_{i=1}^{h} \hat{\beta}_{n_{i}}^{c}$.

6. The simulated bias of $\hat{\beta}_{N}^{c}$ is shown by $\hat{B}\left(\hat{\beta}_{N}^{c}, \beta\right)$ and its formula is $\hat{E}\left(\hat{\beta}_{N}^{c}\right)-\beta$.

7. The simulated risk of $\hat{\beta}_{N}^{c}$ is shown by $\hat{R}\left(\hat{\beta}_{N}^{c}, \beta\right)$ and its formula is $\bar{r}^{c}=A h^{-1} \sum_{i=1}^{h}\left(\hat{\beta}_{n_{i}}^{c}-\beta\right)^{2}$.

In Table 1 , we present the results with $\beta=5$. Table 2 shows the results when $\omega$ expressed as a function of $\beta$. Fig. 1(a) compares $\hat{R}\left(\hat{\beta}_{N}, \beta\right)$ and $\hat{R}\left(\hat{\beta}_{N}^{c}, \beta\right)$ for different values of $\beta$. Also, Fig. 1(b) compares $\hat{B}\left(\hat{\beta}_{N}, \beta\right)$ and $\hat{B}\left(\beta_{N}^{c}, \beta\right)$ for different values of $\beta$.

(a) The curves of $\widehat{R}\left(\widehat{\beta}_{N}, \beta\right)$ and $\widehat{R}\left(\widehat{\beta}_{N}^{c}, \beta\right)$ as functions of $\beta$

(b) The curves of $\widehat{\operatorname{Bias}}\left(\hat{\beta}_{N}, \beta\right)$ and $\widehat{\operatorname{Bias}}\left(\hat{\beta}_{N}^{c}, \beta\right)$ as functions of $\beta$
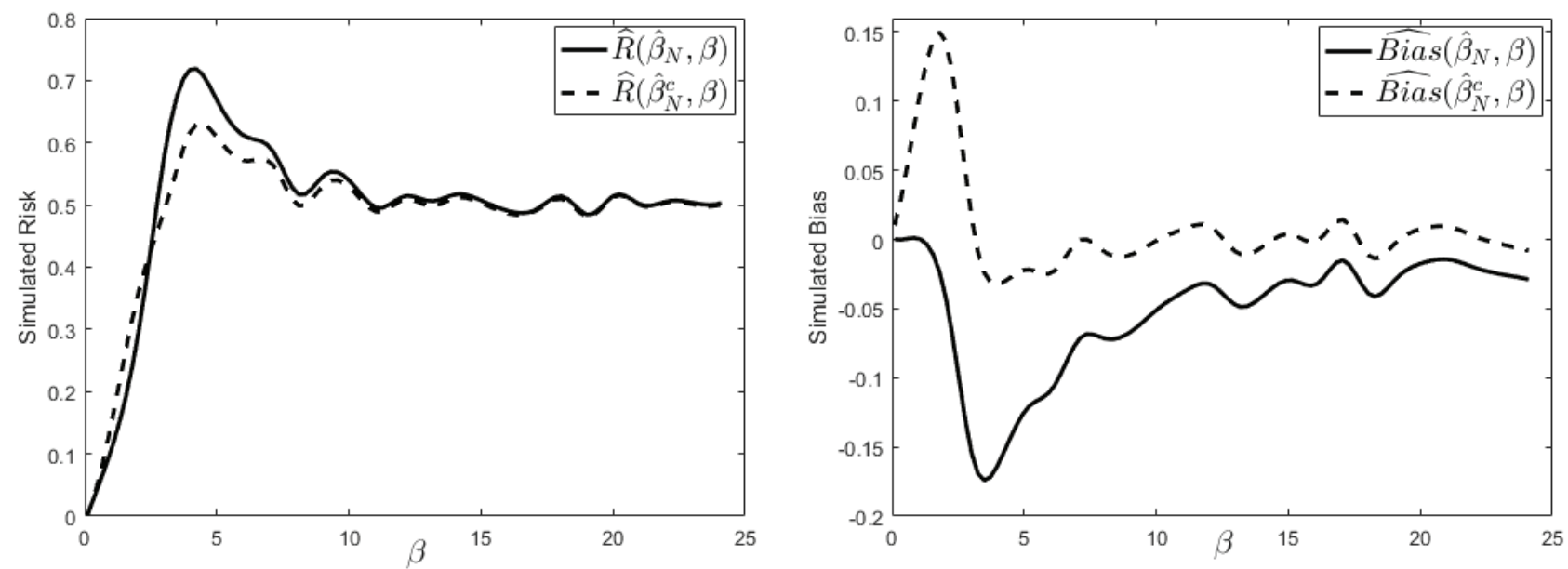

Figure 1 The curves of simulated risk and bias as functions of $\beta$, for $\alpha=1, A=2, m=20$ and $\omega=0.5$.

Some of important observed features of simulation results are as follows:

1. As we expect, the exact and estimated values of $E(N)$ are less than $n^{*}$.

2. The negativity of $B\left(\hat{\beta}_{N}, \beta\right)$ is verified.

3. The smaller value of $\omega$, the better estimate of $\beta$.

4. The larger value of $\alpha$, the better estimate of $\beta$ and the smaller final sample size.

5. As we expect by Theorem 3.3, when the parameter $\beta$ increases then $\hat{B}\left(\hat{\beta}_{N}, \beta\right)$ decreases.

6. When $\beta$ is small, $\hat{R}\left(\hat{\beta}_{N}, \beta\right)$, simulated risk, is less than $\omega$, but this is not true at all and for some values $\beta$, we have $\hat{R}\left(\hat{\beta}_{N}, \beta\right)>\omega$.

7. When $\beta$ is increasing to large values, the risk decreases and converges to $\omega$.

8. As we expect, $\hat{B}\left(\hat{\beta}_{N}^{c}, \beta\right)$ is less than $\hat{B}\left(\hat{\beta}_{N}, \beta\right)$ and this verifies that, $\hat{\beta}_{N}^{c}$ is better than $\hat{\beta}_{N}$ as an estimator of $\beta$.

9. $\hat{R}\left(\hat{\beta}_{N}^{c}, \beta\right)$ is less than $\hat{R}\left(\hat{\beta}_{N}^{c}, \beta\right)$, but still for some values $\beta$, the risk of $\hat{\beta}_{N}^{c}$ is greater than $\omega$.

10. As we see, the two ratio $\hat{R}\left(\hat{\beta}_{N}, \beta\right) / \hat{R}\left(\hat{\beta}_{n^{*}}, \beta\right)$ and $\hat{R}\left(\hat{\beta}_{N}^{c}, \beta\right) / \hat{R}\left(\hat{\beta}_{n^{*}}, \beta\right)$ converge to 1 as $w \rightarrow 0$.

11. The second order asymptotic efficiency result, $E(N)-n^{*}=O(1)$, is seen in tables. 
Table 1 Simulations of purely sequential procedure under gamma distribution.

\begin{tabular}{|c|c|c|c|c|c|c|}
\hline \multicolumn{7}{|c|}{$m=5, A=2, \beta=5, \alpha=1$} \\
\hline$\omega$ & 2.000 & 1.000 & 0.500 & 0.250 & 0.100 & 0.050 \\
\hline$n^{*}$ & 25.000 & 50.000 & 100.000 & 200.000 & 500.000 & 1000.000 \\
\hline$E(N)$ & 23.068 & 48.068 & 98.068 & 198.068 & 498.068 & 998.068 \\
\hline$\hat{E}(N)$ & 21.872 & 45.896 & 96.401 & 197.400 & 497.440 & 998.222 \\
\hline$E\left(\hat{\beta}_{N}\right)$ & 4.600 & 4.800 & 4.900 & 4.950 & 4.980 & 4.990 \\
\hline$\hat{E}\left(\hat{\beta}_{N}\right)$ & 4.363 & 4.616 & 4.833 & 4.936 & 4.976 & 4.990 \\
\hline$\hat{R}\left(\hat{\beta}_{N}, \beta\right) / R\left(\hat{\beta}_{n}^{*}, \beta\right)$ & 2.160 & 2.522 & 2.016 & 1.427 & 1.202 & 0.993 \\
\hline$\hat{E}\left(\hat{\beta}_{N}^{c}\right)$ & 4.822 & 4.841 & 4.939 & 4.987 & 4.996 & 5.000 \\
\hline$\hat{R}\left(\hat{\beta}_{N}^{c}, \beta\right) / R\left(\hat{\beta}_{n}^{*}, \beta\right)$ & 1.478 & 1.995 & 1.776 & 1.345 & 1.171 & 0.986 \\
\hline \multicolumn{7}{|c|}{$m=10, A=2, \beta=5, \alpha=1$} \\
\hline$\omega$ & 2.000 & 1.000 & 0.500 & 0.250 & 0.100 & 0.050 \\
\hline$n^{*}$ & 25.000 & 50.000 & 100.000 & 200.000 & 500.000 & 1000.000 \\
\hline$E(N)$ & 23.068 & 48.068 & 98.068 & 198.068 & 498.068 & 998.068 \\
\hline$\hat{E}(N)$ & 23.392 & 47.109 & 97.356 & 197.681 & 497.828 & 997.396 \\
\hline$E\left(\hat{\beta}_{N}\right)$ & 4.600 & 4.800 & 4.900 & 4.950 & 4.980 & 4.990 \\
\hline$\hat{E}\left(\hat{\beta}_{N}\right)$ & 4.580 & 4.714 & 4.871 & 4.944 & 4.979 & 4.988 \\
\hline$\hat{R}\left(\hat{\beta}_{N}, \beta\right) / R\left(\hat{\beta}_{n}^{*}, \beta\right)$ & 1.358 & 1.763 & 1.466 & 1.137 & 1.055 & 1.007 \\
\hline$\hat{E}\left(\hat{\beta}_{N}^{c}\right)$ & 5.003 & 4.929 & 4.974 & 4.994 & 4.999 & 4.998 \\
\hline$\hat{R}\left(\hat{\beta}_{N}^{c}, \beta\right) / R\left(\hat{\beta}_{n}^{*}, \beta\right)$ & 1.031 & 1.447 & 1.330 & 1.088 & 1.037 & 0.998 \\
\hline \multicolumn{7}{|c|}{$m=5, A=2, \beta=5, \alpha=3.7$} \\
\hline$\omega$ & 2.000 & 1.000 & 0.500 & 0.250 & 0.100 & 0.010 \\
\hline$n^{*}$ & 6.757 & 13.514 & 27.027 & 54.054 & 135.135 & 270.270 \\
\hline$E(N)$ & 6.762 & 13.519 & 27.032 & 54.059 & 135.140 & 270.275 \\
\hline$\hat{E}(N)$ & 7.289 & 13.447 & 27.032 & 53.950 & 135.219 & 270.293 \\
\hline$E\left(\hat{\beta}_{N}\right)$ & 4.600 & 4.800 & 4.900 & 4.950 & 4.980 & 4.990 \\
\hline$\hat{E}\left(\hat{\beta}_{N}\right)$ & 4.757 & 4.778 & 4.895 & 4.944 & 4.981 & 4.990 \\
\hline$\hat{R}\left(\hat{\beta}_{N}, \beta\right) / R\left(\hat{\beta}_{n}^{*}, \beta\right)$ & 0.883 & 1.312 & 1.260 & 1.145 & 1.034 & 0.999 \\
\hline$\hat{E}\left(\hat{\beta}_{N}^{c}\right)$ & 5.121 & 4.979 & 4.996 & 4.994 & 5.001 & 5.000 \\
\hline$\hat{R}\left(\hat{\beta}_{N}^{c}, \beta\right) / R\left(\hat{\beta}_{n}^{*}, \beta\right)$ & 0.795 & 1.120 & 1.163 & 1.097 & 1.019 & 0.991 \\
\hline
\end{tabular}

Table 2 Simulations of purely sequential procedure under gamma distribution when $\omega$ expressed as a function of $\beta$.

\begin{tabular}{lcccc}
\hline \multicolumn{5}{c}{$\boldsymbol{m}=\mathbf{5}, \boldsymbol{A}=\mathbf{2}, \boldsymbol{\beta}=\mathbf{0 . 5}, \boldsymbol{\alpha}=1$} \\
\hline$\omega$ & $0.1 \beta$ & $0.05 \beta$ & $0.025 \beta$ & $0.001 \beta$ \\
$n^{*}$ & 10.000 & 20.000 & 40.000 & 1000.000 \\
$E(N)$ & 8.068 & 18.068 & 38.068 & 998.068 \\
$\hat{E}(N)$ & 9.652 & 17.604 & 36.142 & 998.592 \\
$E\left(\hat{\beta}_{N}\right)$ & 0.400 & 0.450 & 0.475 & 0.499 \\
$\hat{E}\left(\hat{\beta}_{N}\right)$ & 0.432 & 0.433 & 0.454 & 0.499 \\
$\hat{R}\left(\hat{\beta}_{N}, \beta\right) / R\left(\hat{\beta}_{n}^{*}, \boldsymbol{\beta}\right)$ & 1.022 & 1.821 & 2.424 & 0.998 \\
$\hat{E}\left(\hat{\beta}_{N}^{c}\right)$ & 0.528 & 0.489 & 0.482 & 0.500 \\
$\hat{R}\left(\hat{\beta}_{N}^{c}, \beta\right) / R\left(\hat{\beta}_{n}^{*}, \boldsymbol{\beta}\right)$ & 0.795 & 1.216 & 1.839 & 0.991 \\
\hline
\end{tabular}




\section{PURELY SEQUENTIAL PROCEDURE USING ADJUSTED $\boldsymbol{N}$}

Recall that we want to introduce an estimator of parameter $\beta$ such that its associated risk is not greater than $\omega$, but $\hat{\beta}_{N}$ and $\hat{\beta}_{N}^{c}$ did not have this property. Since the stopping time defined by (3) underestimates $n^{*}$, we believe that by adjusting $N$ this problem can be solved.

To avoid underestimation at the termination, we consider the following stopping time

$$
N_{a d j}=\inf \left\{n \geq m ; S_{n}<\sqrt{\frac{\alpha^{3} \omega}{A}} n^{1.5} L_{n}\right\}
$$

where $L_{n}=1+\frac{L_{0}}{n}+o\left(\frac{1}{n}\right)$ as $n \rightarrow \infty$ and $L_{n}>1$ for any $n$. This stopping time is the adjusted version of (3), and is obtained by adding $L_{n}$ to the right hand side of (3). Now, we can use (8) as a stopping time and run a purely sequential procedure. As soon as sampling terminate with this procedure, we define $\hat{\beta}_{N_{a d j}}=\frac{X_{1}+X_{2}+\cdots+X_{N_{a d j}}}{\alpha N_{a d j}}=\frac{\bar{X}_{N_{a d j}}}{\alpha}$ as an estimator of $\beta$. We will see that $\hat{\beta}_{N_{a d j}}$ is a bias estimator of $\beta$.

Theorem 5.1. (The first moment of $N_{a d j}$ ) Suppose that $m \alpha>2$ and $r>2$. If $n^{*}>m$ then

$$
\begin{aligned}
E\left(N_{a d j}\right)= & n^{*}-\frac{1}{\alpha}+\frac{1}{2}+L_{0}-2 \sum_{n=1}^{\infty}\left[\bar{G}\left(\frac{3}{2} n \alpha, n \alpha+1,1\right)\right. \\
& \left.-\frac{3}{2} \bar{G}\left(\frac{3}{2} n \alpha, n \alpha, 1\right)\right]+o(1),
\end{aligned}
$$

as $\omega \rightarrow 0$.

Proof. To proof this theorem, we use Theorem 2.4 of Woodroofe [1] (see Appendix).

After comparing $N_{a d j}$ with the stopping time in Theorem 2.4 of Woodroofe [1], we have $a=\frac{3}{2}, b=\frac{1}{a-1}=2, \mu=\alpha \beta, \tau^{2}=\alpha \beta^{2}$, $c=\sqrt{\frac{\alpha^{3} \omega}{A}}$ and $\lambda=\frac{\mu^{b}}{c^{b}}=\frac{A \beta^{2}}{\alpha \omega}=n^{*}$.

First, note that

$$
F(x)=\int_{0}^{x} \frac{t^{\alpha-1} e^{-t / \beta}}{\beta^{\alpha} \Gamma(\alpha)} d t \leq \frac{1}{\beta^{\alpha} \Gamma(\alpha)} \int_{0}^{x} t^{\alpha-1} d t=\frac{x^{\alpha}}{\beta^{\alpha} \Gamma(\alpha+1)}
$$

Thus, putting $p=\alpha$ and $B=\frac{1}{\beta^{\alpha} \Gamma(\alpha+1)}$, the conditions are hold. Also, $E\left[X^{r}\right]<\infty$ for all $r>2$.

To obtain $\nu$, we must compute $E\left\{\left(S_{n}-n a \mu\right)^{+}\right\}$. Since $X_{i} \sim \operatorname{Gamma}(\alpha, \beta)$, we know that $\frac{S_{n}}{\beta} \sim \operatorname{Gamma}(n \alpha, 1)$. Hence,

$$
\begin{aligned}
E\left[\left(S_{n}-n a \mu\right)^{+}\right] & =E\left[\left(S_{n}-\frac{3}{2} n \alpha \beta\right)^{+}\right] \\
& =\beta E\left[\left(\frac{S_{n}}{\beta}-\frac{3}{2} n \alpha\right)^{+}\right] \\
& =\beta \int_{\frac{3}{2} n \alpha}^{\infty}\left(t-\frac{3}{2} n \alpha\right) \frac{1}{\Gamma(n \alpha)} t^{n \alpha-1} e^{-t} d t \\
& =n \alpha \beta\left[\bar{G}\left(\frac{3}{2} n \alpha, n \alpha+1,1\right)-\bar{G}\left(\frac{3}{2} n \alpha, n \alpha, 1\right)\right] .
\end{aligned}
$$

Thus, to obtain $\nu$ we have

$$
\begin{aligned}
\nu & =\frac{b}{2 \mu}\left[(a-1)^{2} \mu^{2}+\tau^{2}\right]-\sum_{n=1}^{\infty} \frac{1}{n} E\left\{\left(S_{n}-n a \mu\right)^{+}\right\} \\
& =\alpha \beta\left\{\frac{1}{4}+\frac{1}{\alpha}-\sum_{n=1}^{\infty}\left[\bar{G}\left(\frac{3}{2} n \alpha, n \alpha+1,1\right)-\bar{G}\left(\frac{3}{2} n \alpha, n \alpha, 1\right)\right]\right\} .
\end{aligned}
$$


Finally, by substituting Eq. (11), $a=\frac{3}{2}, b=2, \mu=\alpha \beta$ and $\tau^{2}=\alpha \beta^{2}$ into (14), the proof of this theorem is completed.

Theorem 5.2. If $m \alpha>2$, then the expectation of $\hat{\beta}_{N_{a d j}}$ is

$$
E\left(\hat{\beta}_{N_{a d j}}\right)=\beta-\frac{2 \beta}{\alpha n^{*}}+o(1)
$$

as $\omega \rightarrow 0$.

Proof. If we rewrite $N_{a d j}$ as

$$
N_{a d j}=\inf \left\{n \geq m ; \frac{n}{\frac{L_{n} \bar{X}_{n}^{2}}{\alpha^{2} \beta^{2}}}>n^{*}\right\}=\inf \left\{n \geq m ; \frac{n}{L_{n} \bar{Y}_{n}^{2}}>n^{*}\right\}=\inf \left\{n \geq m ; Z_{n}>n^{*}\right\},
$$

where $Y_{i}=\frac{X_{i}}{\alpha \beta}$ and $Z_{n}=\frac{n}{L_{n} \bar{Y}_{n}^{2}}$, the proof of this theorem is the same as proof of Theorem 2 of Takada and Nagata [2], thus we ignore to give the details.

By Theorem 5.2, we can correct the bias and introduce a corrected estimator as follows:

$$
\hat{\beta}_{N_{a d j}}^{c}=\frac{\bar{X}_{N_{a d j}}}{\alpha}\left(1+\frac{2}{\alpha N_{a d j}}\right)
$$

The bias of this estimator is negligible and hence, is better than (12).

To find an appropriate $N_{a d j}$, we have considered $\alpha=1, L_{n}=1+\frac{L_{0}}{n}+o\left(\frac{1}{n}\right)$ and by empirical investigations saw that if we let $L_{0}=20$ then we get the satisfactory results. Unfortunately, attempts to calculate the risk analytically has not let to a conclusion. Therefore, to resolve this captivity and to select a suitable value for $L_{0}$, we use the simulation and empirical results with different values of the parameters involved in the model. The result of these extensive studies shows that the most appropriate value for $L_{0}$ is 20 .

Table 3 shows the exact and simulated values of $E\left(N_{a d j}\right), E\left(\hat{\beta}_{N_{a d j}}\right), \hat{R}\left(\hat{\beta}_{N_{a d j}}, \beta\right), \hat{E}\left(\hat{\beta}_{N_{a d j}}^{c}\right)$ and $\hat{R}\left(\hat{\beta}_{N_{a d j}}^{c}, \beta\right)$. Table 4 shows the results with adjusted $N$ when $\omega$ expressed as a function of $\beta$. Also, Fig. 2 shows the curves of simulated biases and simulated risks as functions of $\beta$. Since we want to compare the results of usual purely sequential procedure and purely sequential procedure with $N_{a d j}$, all of the conditions in two simulations are the same except that here we use adjusted $N$ as stopping time. Hence, one can compare Table 1 with Table 3 , Table 2 with Table 4 and Fig. 1 with Fig. 2 . This compare shows that when we use $N_{a d j}$, the results improve. Also, the simulated results verify

\begin{tabular}{|c|c|c|c|c|c|c|}
\hline \multicolumn{7}{|c|}{$m=5, A=2, \beta=5, \alpha=1$} \\
\hline$\omega$ & 2.000 & 1.000 & 0.500 & 0.250 & 0.100 & 0.050 \\
\hline$n^{*}$ & 25.000 & 50.000 & 100.000 & 200.000 & 500.000 & 1000.000 \\
\hline$E\left(N_{a d j}\right)$ & 43.068 & 68.068 & 118.068 & 218.068 & 518.068 & 1018.068 \\
\hline$\hat{E}\left(N_{a d j}\right)$ & 36.996 & 63.841 & 115.344 & 216.719 & 517.556 & 1017.715 \\
\hline$E\left(\hat{\beta}_{N_{a d j}}\right)$ & 4.600 & 4.800 & 4.900 & 4.950 & 4.980 & 4.990 \\
\hline$\hat{E}\left(\hat{\beta}_{N_{a d j}}\right)$ & 4.754 & 4.844 & 4.911 & 4.955 & 4.981 & 4.990 \\
\hline$\hat{R}\left(\hat{\beta}_{N_{a d j}}, \beta\right) / R\left(\hat{\beta}_{n}^{*}, \beta\right)$ & 0.977 & 1.004 & 0.986 & 1.007 & 0.986 & 0.984 \\
\hline$\hat{E}\left(\hat{\beta}_{N_{a d j}}^{c}\right)$ & 5.017 & 4.999 & 4.997 & 5.001 & 5.001 & 5.000 \\
\hline$\hat{R}\left(\hat{\beta}_{N_{a d j}}^{c}, \beta\right) / R\left(\hat{\beta}_{n}^{*}, \beta\right)$ & 0.874 & 0.918 & 0.930 & 0.976 & 0.973 & 0.977 \\
\hline
\end{tabular}
the superiority of $\hat{\beta}_{N_{a d j}}^{c}$.

Table 3 Simulations of purely sequential procedure with adjusted $N$ under the gamma distribution. 


\begin{tabular}{|c|c|c|c|c|c|c|}
\hline \multicolumn{7}{|c|}{$m=10, A=2, \beta=5, \alpha=1$} \\
\hline$\omega$ & 2.000 & 1.000 & 0.500 & 0.250 & 0.100 & 0.050 \\
\hline$n^{*}$ & 25.000 & 50.000 & 100.000 & 200.000 & 500.000 & 1000.000 \\
\hline$E\left(N_{\text {adj }}\right)$ & 43.068 & 68.068 & 118.068 & 218.068 & 518.068 & 1018.068 \\
\hline$\hat{E}\left(N_{a d j}\right)$ & 36.793 & 63.994 & 115.624 & 216.735 & 517.910 & 1018.271 \\
\hline$E\left(\hat{\beta}_{N_{a d j}}\right)$ & 4.600 & 4.800 & 4.900 & 4.950 & 4.980 & 4.990 \\
\hline$\hat{E}\left(\hat{\beta}_{N_{a d j}}\right)$ & 4.741 & 4.853 & 4.918 & 4.956 & 4.983 & 4.992 \\
\hline$\hat{R}\left(\hat{\beta}_{N_{a d j}}, \beta\right) / R\left(\hat{\beta}_{n}^{*}, \beta\right)$ & 0.894 & 0.963 & 0.960 & 0.992 & 0.977 & 0.977 \\
\hline$\hat{E}\left(\hat{\beta}_{N_{a d j}}^{c}\right)$ & 5.004 & 5.008 & 5.004 & 5.002 & 5.003 & 5.001 \\
\hline$\hat{R}\left(\hat{\beta}_{N_{a d j}}^{c}, \beta\right) / R\left(\hat{\beta}_{n}^{*}, \beta\right)$ & 0.787 & 0.884 & 0.909 & 0.961 & 0.965 & 0.970 \\
\hline \multicolumn{7}{|c|}{$m=5, A=2, \beta=5, \alpha=3.7$} \\
\hline$\omega$ & 2.000 & 1.000 & 0.500 & 0.250 & 0.100 & 0.050 \\
\hline$n^{*}$ & 6.757 & 13.514 & 27.027 & 54.054 & 135.135 & 270.270 \\
\hline$E\left(N_{a d j}\right)$ & 26.762 & 33.519 & 47.032 & 74.059 & 155.140 & 290.275 \\
\hline$\hat{E}\left(N_{a d j}\right)$ & 15.765 & 24.804 & 40.619 & 69.855 & 152.920 & 289.025 \\
\hline$E\left(\hat{\beta}_{N_{a d j}}\right)$ & 4.600 & 4.800 & 4.900 & 4.950 & 4.980 & 4.990 \\
\hline$\hat{E}\left(\hat{\beta}_{N_{a d j}}\right)$ & 4.883 & 4.923 & 4.946 & 4.971 & 4.983 & 4.991 \\
\hline$\hat{R}\left(\hat{\beta}_{N_{a d j}}, \beta\right) / R\left(\hat{\beta}_{n}^{*}, \beta\right)$ & 0.481 & 0.593 & 0.695 & 0.790 & 0.912 & 0.949 \\
\hline$\hat{E}\left(\hat{\beta}_{N_{a d j}}^{c}\right)$ & 5.052 & 5.030 & 5.013 & 5.010 & 5.001 & 5.000 \\
\hline$\hat{R}\left(\hat{\beta}_{N_{a d j}}^{c}, \beta\right) / R\left(\hat{\beta}_{n}^{*}, \beta\right)$ & 0.463 & 0.574 & 0.676 & 0.777 & 0.901 & 0.942 \\
\hline
\end{tabular}

Table 4 Simulations of purely sequential procedure with adjusted $\mathrm{N}$ under the gamma distribution when $\omega$ expressed as a function of $\beta$.

\begin{tabular}{|c|c|c|c|c|}
\hline \multicolumn{5}{|c|}{$m=5, A=2, \beta=0.5, \alpha=1$} \\
\hline$\omega$ & $1 \beta$ & $0.05 \beta$ & $0.025 \beta$ & $0.001 \beta$ \\
\hline$n^{*}$ & 10.000 & 20.000 & 40.000 & 1000.000 \\
\hline$E\left(N_{a d j}\right)$ & 28.068 & 38.068 & 58.068 & 1018.068 \\
\hline$\hat{E}\left(N_{a d j}\right)$ & 18.993 & 31.194 & 53.489 & 1018.625 \\
\hline$\left.N_{a d j}\right)$ & 0.400 & 0.450 & 0.475 & 0.499 \\
\hline$\hat{E}\left(\hat{\beta}_{N_{a d j}}\right)$ & 0.456 & 0.470 & 0.483 & 0.499 \\
\hline$\hat{R}\left(\hat{\beta}_{N_{a d j}}, \beta\right) / R\left(\hat{\beta}_{n}^{*}, \beta\right)$ & 0.748 & 0.939 & 0.986 & 0.988 \\
\hline$\hat{E}\left(\hat{\beta}_{N_{a d j}}^{c}\right)$ & 0.505 & 0.501 & 0.501 & 0.500 \\
\hline$\hat{R}\left(\hat{\beta}_{N_{a d j}}^{c}, \beta\right) / R\left(\hat{\beta}_{n}^{*}, \beta\right)$ & 0.641 & 0.827 & 0.901 & 0.982 \\
\hline
\end{tabular}

\section{COMPARING PURELY SEQUENTIAL AND TWO-STAGE PROCEDURES}

The two-stage procedure for estimating the scale parameter of a gamma distribution was introduced by Mahmoudi and Roughani [5]. The most important weakness of this procedure is oversampling. To reduce this weakness, the two-stage procedure was improved by Roughani and Mahmoudi [6], but the oversampling problem was not solved utterly. The purely sequential procedure reduces oversampling. Here, we compare the results of the improved two-stage procedure (two-stage procedure with $B^{\text {new }}$ ) and the results of the purely sequential procedure. Table 5 shows the simulation results. The results show that for small values of $\omega$, final sample size of purely sequential procedure is less than final sample size of two-stage procedure and this difference in size of samples is bolder for small values of $\alpha$. In sequential estimation problem, we may expect risk efficiency to lie in a close proximity of one if $\omega$ tend to zero, so simulated risk efficiency is shown in Fig. 3. 
(a) The curves of $\widehat{R}\left(\widehat{\beta}_{N_{a d j}}, \beta\right)$ and $\widehat{R}\left(\widehat{\beta}_{N_{a d j}}^{c}, \beta\right)$ as functions of $\beta$

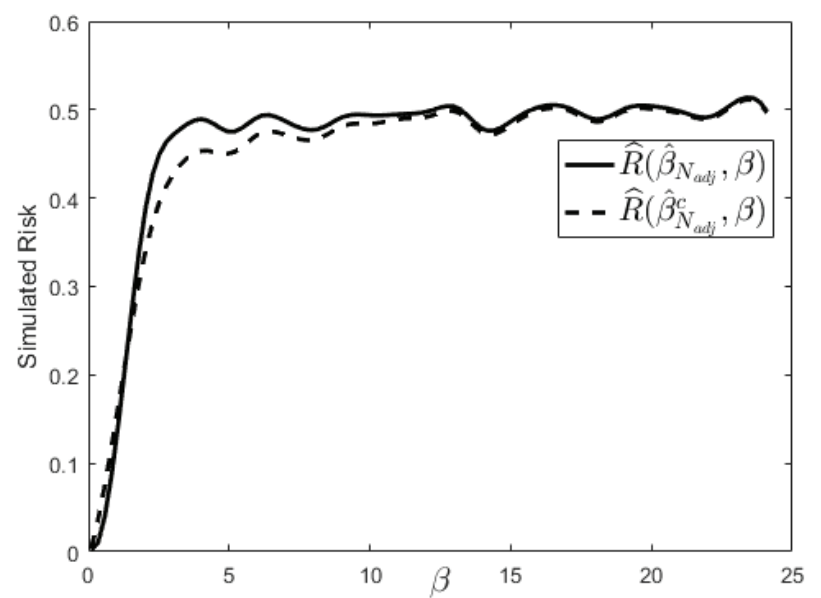

(b) The curves of $\widehat{\operatorname{Bias}}\left(\hat{\beta}_{N_{a d j}}, \beta\right)$ and $\widehat{\operatorname{Bias}}\left(\hat{\beta}_{N_{a d j}}^{c}, \beta\right)$ as functions of $\beta$

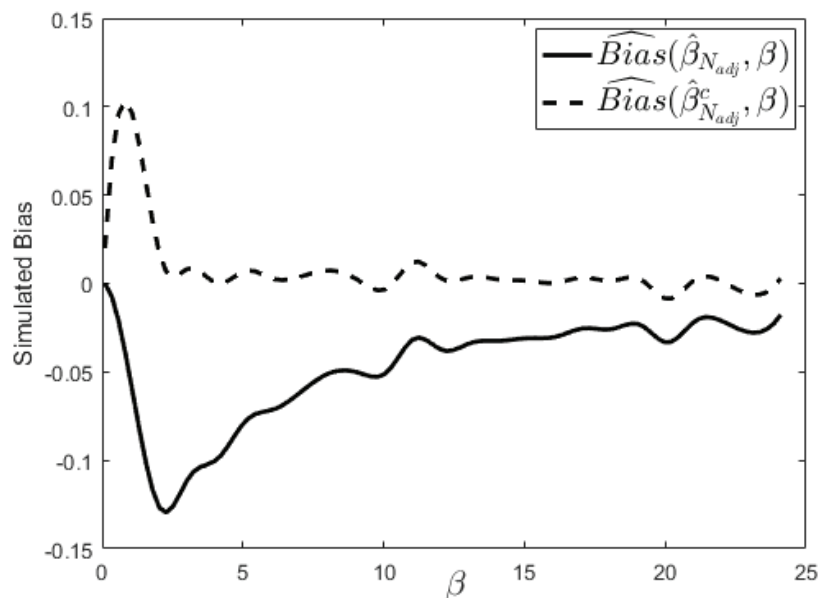

Figure 2 The curves of simulated risk and bias as functions of $\beta$ for $\alpha=1, A=2, m=20$ and $\omega=0.5$ with adjusted N.

(a) The curves of $\widehat{R}\left(\widehat{\beta}_{N}, \beta\right) / R\left(\widehat{\beta}_{n^{*}}, \beta\right)$ and $\widehat{R}\left(\widehat{\beta}_{N}^{c}, \beta\right) / R\left(\widehat{\beta}_{n^{*}}, \beta\right)$.

(b) The curves of $\widehat{R}\left(\widehat{\beta}_{N_{a d j}}, \beta\right) / R\left(\widehat{\beta}_{n^{*}}, \beta\right)$ and $\widehat{R}\left(\widehat{\beta}_{N_{a d j}}^{c}, \beta\right) / R\left(\widehat{\beta}_{n^{*}}, \beta\right)$
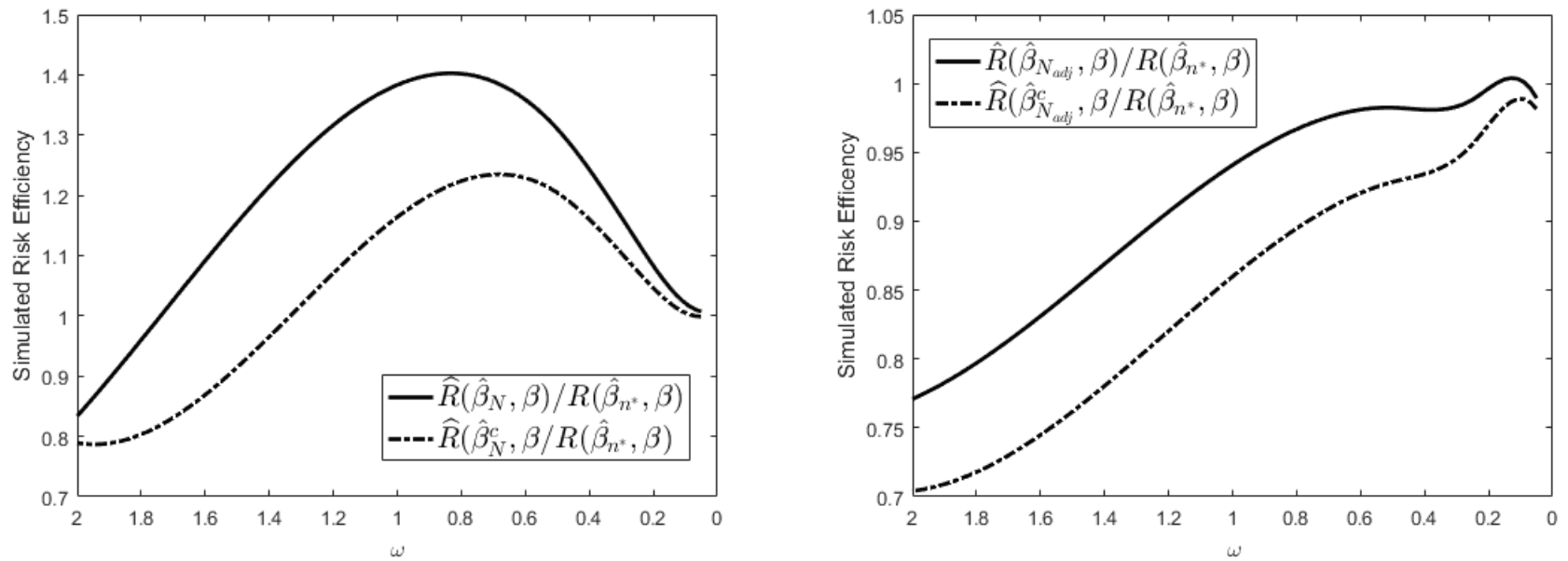

Figure 3 Simulated risk efficiency as a function of $\omega$ for $\alpha=1, A=2, m=20$.

Table 5 Comparing the results of purely sequential and two-stage procedures.

\begin{tabular}{|c|c|c|c|c|c|c|c|}
\hline \multicolumn{8}{|c|}{$m=20, A=2, \beta=5, \alpha=1$} \\
\hline & $\omega$ & 2.0000 & 1.0000 & 0.5000 & 0.2500 & 0.1000 & 0.0500 \\
\hline & $n^{*}$ & 25.000 & 50.000 & 100.00 & 200.00 & 500.00 & 1000.0 \\
\hline \multirow[t]{3}{*}{ Purely sequential } & $\hat{E}\left(N_{a d j}\right)$ & 37.470 & 63.829 & 115.85 & 216.76 & 517.66 & 1018.5 \\
\hline & $\hat{E}\left(\hat{\beta}_{N_{a d j}}\right)$ & 4.8038 & 4.8465 & 4.9236 & 4.9555 & 4.9820 & 4.9921 \\
\hline & $\hat{R}\left(\hat{\beta}_{N_{a d j}}, \beta\right)$ & 1.4925 & 0.9413 & 0.4784 & 0.2549 & 0.0982 & 0.0505 \\
\hline \multirow[t]{3}{*}{ Two-stage } & $\hat{E}\left(N_{m}\right)$ & 37.461 & 73.431 & 145.87 & 292.39 & 729.96 & 1457.8 \\
\hline & $\hat{E}\left(\hat{\beta}_{N_{m}}\right)$ & 4.7558 & 4.8372 & 4.9157 & 4.9568 & 4.9839 & 4.9916 \\
\hline & $\hat{R}\left(\hat{\beta}_{N_{m}}, \beta\right)$ & 1.5970 & 0.9782 & 0.4732 & 0.2240 & 0.0861 & 0.0427 \\
\hline
\end{tabular}




\begin{tabular}{|c|c|c|c|c|c|}
\hline \multicolumn{6}{|c|}{$m=20, A=2, \beta=5, \alpha=3.7$} \\
\hline & $\omega$ & 0.5000 & 0.2500 & 0.1000 & 0.0500 \\
\hline & $n^{*}$ & 27.027 & 54.054 & 135.14 & 270.27 \\
\hline \multirow[t]{3}{*}{ Purely sequential } & $\hat{E}\left(N_{a d j}\right)$ & 40.692 & 69.846 & 153.12 & 289.21 \\
\hline & $\hat{E}\left(\hat{\beta}_{N_{a d j}}\right)$ & 4.9506 & 4.9700 & 4.9871 & 4.9925 \\
\hline & $\hat{R}\left(\hat{\beta}_{N_{a d j}}, \beta\right)$ & 0.3544 & 0.1981 & 0.0913 & 0.0473 \\
\hline \multirow[t]{3}{*}{ Two-stage } & $\hat{E}\left(N_{m}\right)$ & 31.469 & 62.191 & 155.07 & 309.30 \\
\hline & $\hat{E}\left(\hat{\beta}_{N_{m}}\right)$ & 4.9177 & 4.9547 & 4.9815 & 4.9914 \\
\hline & $\hat{R}\left(\hat{\beta}_{N_{m}}, \beta\right)$ & 0.4778 & 0.2426 & 0.0937 & 0.0465 \\
\hline
\end{tabular}

\section{REAL DATA}

Zacks and Khan [3] provided a method to produce a random sample from gamma distribution using a random sample from normal distribution. This paper states that if $Y_{1}, \cdots, Y_{n}$ is a random sample from a normal distribution with mean $\mu$ and variance $\sigma^{2}$, then the transformation $\frac{i-1}{i}\left(Y_{i}-\bar{Y}_{i-1}\right)^{2}, i>2$ gives a random sample of size $n-1$ from gamma distribution with $\alpha=1 / 2$ and $\beta=2 \sigma^{2}$.

In this section, we consider a dataset that consists of 346 observations of weights of babies born between September 2014, 23, and November 2014, 21, in Imam Ali hospital of Shahrekord in Iran. This dataset is given in Roughani and Mahmoudi [6]. This dataset is used to obtain real data from gamma population with known shape parameter $\alpha=1 / 2$ and scale parameter $\beta=2 \sigma^{2}$. The mean and variance for the full data are 3.147 and $0.198 \mathrm{~kg}$, respectively. The associated P-values according to goodness of fit test for normal and gamma distributions are 0.29 and 0.40 , which made us feel reasonably assured of an underlying gamma distribution for transmuted dataset.

Treating transformed data set as the universe, we implemented purely sequential procedure, drawing observations from the full set of data as needed. Table 6 provides the results derived from implementing the stopping rules from (3), when the initial sample size $m=5,10,20$. $25, \omega=0.03$ and $A=2$ are chosen arbitrarily. Under purely sequential procedure, the final estimators $\hat{\beta}_{N}$ tended to get closer to its true value $\hat{\beta}=0.396$ obtained from full data.

Table 6 An illustration of purely procedure with transformed data, when $m=5,10,20,25, \omega=0.03$ and $A=2$.

$m=5$

Pilot Data:

$0.0658,0.6411,0.0903,0.0162,0.0316$

$\hat{\beta}_{m}=0.338$

Samples:

$0.0515,0.4734,0.3122,0.0681,0.4683$,

$0.0652,0.1088,0.0001,0.0932,0.0361$,

0.1117

$\rightarrow N=16 \quad \hat{\beta}_{N}=0.3292$

$m=10$

Pilot Data:

1.1970, 0.1248, 0.0003, 0.0658, 0.0349,

$0.4524,0.0001,0.0149,0.1679,0.0785$

$\hat{\beta}_{m}=0.4273$

Samples:

$0.0001,0.2090,0.3122,0.1122,0.0227$,

$0.0676,0.1104$

$\rightarrow N=17 \quad \hat{\beta}_{N}=0.3495$ 
Table 6 An illustration of purely procedure with transformed data, when $m=5,10,20,25, \omega=0.03$ and $A=2$. (Continued)

\begin{tabular}{l}
\hline $\boldsymbol{m}=\mathbf{2 0}$ \\
\hline Pilot Data: \\
$0.5607,0.0800,0.2105,0.0785,0.0203$, \\
$0.7156,0.1850,0.0928,0.5967,0.0515$, \\
$0.5607,0.1520,0.1693,0.0618,0.0001$, \\
$0.1537,0.2652,0.1090,0.0073,0.1088$ \\
$\hat{\beta}_{m}=0.4180$ \\
Samples: \\
$0.3907,0.1679,0.3907,0.0020,0.0336$ \\
$\rightarrow N=25 \quad \hat{\beta}_{N}=0.4131$ \\
\hline $\boldsymbol{m}=25$ \\
\hline Pilot Data: \\
$0.2044,0.0958,0.0361,0.3223,0.0001$, \\
$0.1112,0.0553,1.5112,0.1653,0.0289$, \\
$0.1245,0.1445,0.4910,0.0534,0.0252$, \\
$0.2466,0.0252,0.0443,0.0275,0.1319$, \\
$0.3227,0.2161,0.0581,0.2518,0.0580$ \\
Samples: initial samples is enough \\
$\rightarrow N=25 \quad \hat{\beta}_{N}=0.3801$ \\
\hline
\end{tabular}

\section{CONCLUSION}

Purely sequential sampling procedure is developed for estimating the scale parameter $\beta$ in a gamma distribution with known shape parameter $\alpha$. In this problem, the risk of an estimator $\hat{\beta}$ of $\beta$ is less than from a preassigned number $\omega(>0)$, i.e., $R(\hat{\beta}, \beta)=A E_{\beta}\left\{(\hat{\beta}-\beta)^{2}\right\} \leq \omega$, where $0<A<\infty$ is known. We compute asymptotic expressions for $E[N], E\left[\hat{\beta}_{N}\right]$ and the bias of $\hat{\beta}_{N}$. Also, we propose how to obtain the adjusted stopping variable $N_{a d j}$ so that the risk is uniformly bounded by a preassigned value $\omega$. In the end, the performances of the proposed methodology are investigated with the help of simulations.

The problem of sequential estimation of scale parameter $\beta$ where $\alpha$ is unknown is not easy to solve since the Maximum Likelihood Estimator (MLE) of $\alpha$ in a fixed sample size case, has not the explicit form. On the other hand, if one tries to use moment estimators of $\alpha$ and $\beta$, the problem is so hard because of the complexity of moments estimators of $\alpha$ and $\beta$. Recently, I and my colleagues start a work where the shape parameter $\alpha$ is not known, but very explicit formulas that obtain for known $\alpha$ can not be obtain in the case of unknown $\alpha$.

\section{ACKNOWLEDGMENTS}

The authors thank the anonymous referees for their valuable comments and careful reading, which led to an improvement of the presentation and results of the article. The authors are grateful to the Editor-in-Chief and Editor for their helpful remarks on improving this manuscript. The authors are also indebted to Yazd University for supporting this research.

\section{REFERENCES}

1. M. Woodroofe, Ann. Stat. 5 (1977), 984-995.

2. Y. Takada, Y. Nagata, J. Stat. Plan. Inference. 44 (1995), 277-289.

3. S. Zacks, A. Khan, Sequential Anal. 30 (2011), 297-307.

4. E. Isogai, C. Uno, Stat. Probab. Lett. 22 (1995), 287-293.

5. E. Mahmoudi, G. Roughani, Sequential Anal. 34 (2015), 25-38.

6. G. Roughani, E. Mahmoudi, Sequential Anal. 34 (2015), 387-405.

7. N. Starr, M. Woodroofe, Ann. Math. Stat. 43 (1972), 1147-1154.

8. N. Mukhopadhyay, Metrika. 27 (1980), 281-284.

9. E. Isogai, C. Uno, Ann. Inst. Stat. Math. 46 (1994), 77-82.

10. N. Mukhopadhyay, Ann. Inst. Stat. Math. 46 (1994), 509-519.

11. N. Mukhopadhyay, S. Datta, S. Afr. Stat. J. 29 (1995), 9-27. https://hdl.handle.net/10520/AJA0038271X_340 
12. C. Uno, E. Isogai, D.L. Lim, Austrian J. Stat. 33 (2004), 281-291.

13. S. Zacks, N. Mukhopadhyay, Sequential Anal. 25 (2006), 437-452.

14. S. Zacks, N. Mukhopadhyay, Sequential Anal. 25 (2006), 203-226.

15. N. Mukhopadhyay, W. Pepe, Sequential Anal. 25 (2006), 85-101.

16. S. Zacks, Sequential Anal. 28 (2009), 69-81.

17. E. Mahmoudi, R. Lalehzari, Sequential Anal. 36 (2017), 38-54.

18. R. Lalehzari, E. Mahmoudi, A. Khalifeh, Sequential Anal. 37 (2018), 293-310.

19. C. Amero, M.J. Bayarri, J. Stat. Plan. Inference. 58 (1997), 241-261.

20. J. Choe, N. Shroff, in Proceeding of Sixteenth Annual Joint Conference of the IEEE Computer and Communications Societies, Washington, 1997, pp. 549-554.

21. R. Killick, I. Eckley, J. Stat. Softw. 58 (2014), 1-19.

22. J. Dopke, Int. J. Qual. Reliab. Manag. 11 (1994), 27-43.

23. D. Coit, T. Jin, IIE Trans. 32 (2000), 1161-1166.

24. A. Mareuil, R. Leconte, F. Brissette, M. Minville, Can. J. Civ. Eng. 34 (2007), 1048-1060.

25. Y. Takada, Sequential Anal. 5 (1986), 93-101. 


\section{APPENDIX}

Theorem 2.4 of Woodroofe [1]:

Suppose $X_{1}, X_{2}, \cdots$ is a sequence of positive i.i.d random variables having cumulative distribution function $F(x)$, mean $\mu$ and variance $\tau^{2}$ where $F(x) \leq B x^{p}$ for all $x>0$, some $B$ and $p$. Also, $E\left(X_{1}^{r}\right)<\infty$ for some $r$. Let the stopping time be defined as

$$
t_{c}=\inf \left\{n \geq m ; S_{n}<c n^{a} L_{n}\right\}
$$

where $m \geq 1, a>1, S_{n}=\sum_{i=1}^{n} X_{i}$ and $L_{n}=1+\frac{L_{0}}{n}+o\left(\frac{1}{n}\right)$ as $n \rightarrow \infty\left(-\infty<L_{0}<\infty\right)$.

If $r(2 a-1)>4$ and $m p>\frac{1}{a-1}$ then

$$
E\left(t_{c}\right)=\lambda+b \mu^{-1} \nu-b L_{0}-\frac{1}{2} a b^{2} \tau^{2} \mu^{-2}+o(1)
$$

as $c \rightarrow 0$, where $\nu=\frac{b}{2 \mu}\left[(a-1)^{2} \mu^{2}+\tau^{2}\right]-\sum_{n=1}^{\infty} \frac{1}{n} E\left\{\left(S_{n}-n a \mu\right)^{+}\right\}, b=\frac{1}{a-1}$ and $\lambda=\frac{\mu^{b}}{c^{b}}$. 